\title{
QUESTÕES ÉTICAS NA ESCLEROSE MÚLTIPLA SOB O PONTO DE VISTA DE MÉDICOS E PACIENTES
}

\author{
Antonio Paulo Nassar Junior', Daniela Soriano Pignataro', \\ Melissa Martins Fuzaro', Charles Peter Tilbery²
}

RESUMO - A esclerose múltipla (EM) é afecção neurológica que acomete principalmente adultos jovens e evolui, geralmente, para graus variados de incapacidade física dos pacientes. Assim, a abordagem destes pacientes faz com que o médico depare-se com diversas questões éticas. Objetivo: Identificar as percepções de médicos e pacientes sobre a doença e, com isso, melhorar o relacionamento médico-paciente. Método: Foram feitos dois questionários, um respondido por 44 médicos e outro, por 103 pacientes, abordando questões sobre o diagnóstico e a conduta na EM. Resultados: $96,1 \%$ dos pacientes sabiam seu diagnóstico, os outros gostariam de sabê-lo. Daqueles, $74,7 \%$ achavam que a forma contada foi correta e $90,9 \%$ que o médico é que deve contá-lo. Os sintomas que mais os incomodam são a fadiga $(29,1 \%)$ e os déficits motores $(28,1 \%)$. Por outro lado, $68 \%$ dos pacientes afirmaram sofrer com a doença. O motivo mais importante para os médicos contarem o diagnóstico foi para melhorar a adesão ao tratamento $(56,8 \%)$. A presença de um familiar neste momento é exigida por $54,6 \%$ dos médicos. Quando perguntados sobre as orientações de uma gravidez, $50 \%$ dos médicos não responderam adequadamente. Finalmente, $50 \%$ dos médicos manifestaram-se de forma contrária às terapias complementares. Conclusão: Os pacientes querem saber seu diagnóstico e o médico deve contá-lo da forma mais adequada e dar mais informações. Um debate sobre cuidados paliativos também faz-se necessário.

PALAVRAS-CHAVE: esclerose múltipla, ética, relacionamento médico-paciente.

\section{Ethical issues in multiple sclerosis under physicians and patients point of view}

ABSTRACT - Multiple sclerosis (MS) is a neurologic disorder that mostly affects young adults and can usually evolute to physical disability. Thus, caring patients with MS brings many ethic questions for the physician. Objective: To identify physicians and patients' perceptions about the illness and so improve doctor-patient relationship. Method: It was made two different questionnaires, one for patients and another for physicians, 103 patients and 44 physicians answered them. Results: $96.1 \%$ of patients knew their diagnosis, all others would like to know it. From those, $74.7 \%$ thought that that way it was disclosured was correct and $90.9 \%$ said that the doctor should tell us it. The worst symptoms described were fatigue (29.1\%) and motor deficits $(28.1 \%)$. By other side, $68 \%$ of patients told they suffered because of the illness. The most important reason for doctors to tell the diagnosis to the patients was to improve adherence to treatment (56.8\%). A familiar present at this moment was demanded for $54.6 \%$ of doctors. When asked about orientations in a pregnancy, $50 \%$ of physicians did not answer correctly. Finally, $50 \%$ of physicians were against complementary and alternative therapies. Conclusion:Patients want to know their diagnosis and doctors should tell them in the most adequate moment and give more information. A debate about palliative care is also necessary.

KEY WORDS: multiple sclerosis, ethics, doctor-patient relationship.

Muito tem sido falado a respeito da perda do caráter humano da relação médico-paciente. Em parte, ela decorre do próprio avanço da ciência médica, o que obrigou a medicina a dividir-se em especialidades para melhor assimilação e aplicação de seus avanços, mas trouxe como efeito colateral a desper- sonalização de pacientes e médicos ${ }^{1-3}$. $O$ advento da medicina baseada em evidências, posteriormente, embora tenha trazido dados mais fidedignos para a condução das doenças, contribuiu também para que o doente perdesse a sua personalidade ${ }^{4}$. Além da despersonalização que ocorreu, a relação

Disciplina de Neurologia, Departamento de Medicina, Faculdade de Ciências Médicas da Santa Casa de São Paulo, São Paulo, Brasil (FCMSCSP): ' Acadêmico da FCMSCSP; ${ }^{2}$ Professor Adjunto da Disciplina de Neurologia da FCMSCSP Diretor do Centro de Atendimento e Tratamento de Esclerose Múltipla (CATEM). Trabalho agraciado com bolsa de pesquisa do Conselho Regional de Medicina de São Paulo (CREMESP)

Recebido 21 Junho 2004, recebido na forma final 18 Agosto 2004. Aceito 6 Outubro 2004.

Dr. Antonio Paulo Nassar Junior - Avenida Dr. Timóteo Penteado 2690/31 - 07061-000 Guarulhos SP - Brasil. E-mail: paulo_nassar@yahoo.com.br 
médico-paciente também foi alterada no modo como é analisada. O paternalismo de outrora tem dado lugar a uma relação de autonomia de ambas as partes ${ }^{5}$, o que, obviamente, não exime os médicos da responsabilidade por seus atos.

A esclerose múltipla (EM) é doença neurológica caracterizada pela destruição da bainha de mielina no sistema nervoso central, o que resulta em uma série de sintomas motores e sensitivos, manifestados por surtos ${ }^{6}$. A moléstia manifesta-se geralmente no início da idade adulta e, apesar de ter um curso variável, geralmente evolui para a incapacidade física e, conseqüentemente, psicológica do paciente ${ }^{7}$. 0 cuidado de pacientes portadores de EM envolve muitas questões éticas com as quais o neurologista freqüentemente se depara ${ }^{8}$. Estas incluem o momento do diagnóstico, como e quando contá-lo, decisões sobre uma futura gravidez, as conseqüências da progressão da doença e o seu manejo, a participação da família e o impacto do diagnóstico sobre ela, e os cuidados do paciente. Este trabalho pretende orientar o médico que aborda pacientes com EM, incrementando o relacionamento médico-paciente para que os problemas que surgirem com a evolução da doença sejam melhor contornados por eles.

Assim, são objetivos deste estudo: 1. Identificar os sentimentos e o conhecimento dos pacientes portadores de EM acerca da sua doença e, com isso, a primorar o neurologista no relacionamento médicopaciente na EM; 2 . Identificar as percepções que os neurologistas têm das necessidades dos pacientes.

\section{MÉTODO}

Foram entrevistados 103 pacientes do Centro de Atendimento e Tratamento de Esclerose Múltipla (CATEM) da Disciplina de Neurologia da Faculdade de Ciências Médicas da Santa Casa de São Paulo (FCMSESP) e 44 médicos neurologistas, residentes em neurologia do mesmo serviço e dos Hospitais do Servidor Público Estadual, São Paulo (Universidade Federal de São Paulo), Albert Einstein e da Universidade de Mogi das Cruzes, sendo 26 residentes ou estagiários e 18 médicos assistentes.

Os questionários foram realizados com os pacientes, mediante autorização, com termo de consentimento informado, enquanto aguardavam a consulta ou saíam desta no ambulatório. Os questionários dos médicos foram entregues a estes e solicitado que respondessem em um período de tempo pré-determinado. Muitos médicos não responderam o questionário, mas seu número exato não pôde ser determinado.

No questionário dos pacientes, quando se questionou acerca de terapias alternativas, foram incluídas a homeopatia e a acupuntura, pois embora o Conselho Federal de Medicina reconheça as duas como especialidades, esta não é a percepção dos pacientes que responderam ao questionário.

O projeto de pesquisa foi aprovado pelo Comitê de Ética e Pesquisa do Departamento de Medicina da Irmandade da FCMSCSP em 25 de setembro de 2001 e pelo Comitê de Ética em Pesquisa em da ISCMSP em 17 de outubro de 2001 (projeto $n^{\circ}$ 096/01).

Os questionários aplicados a médicos e pacientes encontram-se em poder de um dos autores (APNJ), podendo ser solicitados por correspondência ou e-mail.

\section{RESULTADOS}

Pacientes - Do total de 103 pacientes entrevistados, $88(85,4 \%)$ eram do sexo feminino, com média de idade de 38,4 anos. Quanto ao diagnóstico, a maioria $(96,1 \%)$ o conhecia. Dos quatro pacientes que não o conheciam, todos gostariam de sabê-lo caso o médico o soubesse. Apesar de estarem em um ambulatório especializado, estes quatro pacientes não conheciam seu diagnóstico por ainda estarem em fase de investigação, já que o ambulatório recebe pacientes com suspeita de EM encaminhados do pronto-atendimento do serviço de Neurologia. As opiniões dos pacientes que já tinham conhecimento do seu diagnóstico estão na Tabela 1. Quanto ao segredo, $83(80,6 \%)$ pacientes não se incomodam de que seu diagnóstico seja contado a outras pessoas. Sessenta e nove pacientes $(67,0 \%)$ permitem contar para todos que quiserem saber, $32(31,0 \%)$, apenas íntimos e dois pacientes $(2,0 \%)$ não permitem que o diagnóstico seja contado para ninguém.

$\mathrm{Na}$ amostra feminina, $37(42,5 \%)$ não pretendem ter filhos, $33(37,9 \%)$ já têm e não pretendem ter mais e $17(19,6 \%)$ desejam ter filhos. Em relação

Tabela 1. Respostas acerca do diagnóstico para os pacientes que o sabiam.

\begin{tabular}{lc}
\hline Respostas & Número de pacientes (\%) \\
\hline Forma contada & $74(74,7)$ \\
$\begin{array}{l}\text { Correta } \\
\text { Incorreta }\end{array}$ & $25(25,3)$ \\
Quem deve contar & \\
Médico & $90(90,9)$ \\
Familiar & $4(4,04)$ \\
Médico e familiar & $4(4,04)$ \\
Outros & $1(1,01)$ \\
Quando contar & \\
$\begin{array}{l}\text { Na suspeita clínica } \\
\text { Na confirmação com exames }\end{array}$ & $57(57,8)$ \\
\hline $\begin{array}{l}\text { Fonte: Centro de Atendimento e Tratamento de Esclerose Múltipla (CATEM) } \\
\text { da Disciplina de Neurologia da Faculdade de Ciências Médicas da Santa } \\
\text { Casa de São Paulo. }\end{array}$
\end{tabular}


Tabela 2. Opiniões dos médicos acerca do momento do diagnóstico de esclerose múltipla.

\begin{tabular}{lccc}
\hline Respostas & $\begin{array}{c}\text { Residentes/ } \\
\text { estagiários }(\%)\end{array}$ & $\begin{array}{c}\text { Médicos assistentes } \\
(\%)\end{array}$ & Total $(\%)$ \\
\hline $\begin{array}{l}\text { Motivos para contar } \\
\text { Adesão ao tratamento }\end{array}$ & $13(50,0)$ & $12(66,7)$ & $25(56,8)$ \\
$\begin{array}{l}\text { Direito do paciente } \\
\text { Não respondeu }\end{array}$ & $9(34,6)$ & $4(22,2)$ & $13(29,5)$ \\
Momento de contar & $4(15,4)$ & $2(11,1)$ & $6(13,7)$ \\
Após confirmação & $10(76,9)$ & $15(83,3)$ & $35(79,5)$ \\
Suspeita clínica & $4(15,4)$ & $3(16,7)$ & $7(15,9)$ \\
Outros & $2(7,7)$ & & $2(4,6)$ \\
Presença de familiar & $14(53,8)$ & $10(55,5)$ & $14(31,8)$ \\
Sim & $8(30,8)$ & $6(33,3)$ & $6(13,6)$ \\
Não & $4(15,4)$ & $2(11,1)$ & \\
Às vezes & & & \\
\hline $\begin{array}{l}\text { Fonte: Centro de Atendimento e Tratamento de Esclerose Múltipla (CATEM) da Disciplina de Neurologia } \\
\text { da Faculdade de Ciências Médicas da Santa Casa de São Paulo. }\end{array}$ &
\end{tabular}

Tabela 3. Orientações dos médicos quanto ao tratamento na gravidez.

\begin{tabular}{lccc}
\hline Orientação & $\begin{array}{c}\text { Residentes/ } \\
\text { estagiários (\%) }\end{array}$ & $\begin{array}{c}\text { Médicos } \\
\text { assistentes (\%) }\end{array}$ & Total (\%) \\
\hline Protocolar* & $11(42,3)$ & $11(61,1)$ & $22(50,0)$ \\
Acompanhamento mais constante & $6(23,1)$ & $3(16,7)$ & $9(20,4)$ \\
Piora da doença & $2(7,7)$ & $2(11,1)$ & $4(9,2)$ \\
Não sabe/não respondeu & $7(26,9)$ & $2(11,1)$ & $9(20,4)$ \\
\hline
\end{tabular}

*suspensão do imunossupressor e manutenção do imunomodulador. Fontes: Serviços de Neurologia - Faculdade de Ciências Médicas da Santa Casa de São Paulo, Hospital do Servidor Público Estadual, Universidade Federal de São Paulo, Universidade de Mogi das Cruzes e Hospital Albert Einstein.

\begin{tabular}{lccc}
\multicolumn{1}{l}{ Tabela 4. Opiniões dos médicos acerca do uso de terapias complementares e alternativas. } \\
\hline Opiniões & $\begin{array}{c}\text { Residentes/ } \\
\text { estagiários (\%) }\end{array}$ & $\begin{array}{c}\text { Médicos } \\
\text { assistentes }(\%)\end{array}$ & Total (\%) \\
\hline Sem evidências/indicações & $13(50,0)$ & $9(50,0)$ & $22(50,0)$ \\
Bem-vindas & $5(19,2)$ & $7(38,9)$ & $12(27,3)$ \\
Não sabe/não respondeu & $8(30,8)$ & $2(11,1)$ & $10(22,7)$
\end{tabular}

Fontes: Serviços de Neurologia - Faculdade de Ciências Médicas da Santa Casa de São Paulo, Hospital do Servidor Público Estadual, Universidade Federal de São Paulo, Universidade de Mogi das Cruzes e Hospital Albert Einstein.

à possível hereditariedade da EM, 68 (66,0\%) pacientes negaram que seus filhos pudessem vir a ter a doença, 13 (12,6\%) afirmaram e 22 (21,4\%) não souberam responder.

A distribuição dos sintomas relatados está no Gráfico 1. O sofrimento causado pela doença foi relatado pela maioria dos pacientes $(68,0 \%)$. O sintoma que relatam que mais os incomoda é a fadiga, relatada por 30 pacientes. As sugestões dos pacientes para a melhor condução de seus casos e, conseqüentemente, para sua vida estão no Gráfico 2 . Setenta e cinco pacientes $(72,8 \%)$ disseram não fazer e nem ter feito uso de nenhuma terapia dita alternativa. As terapias mais citadas foram: acupuntura (10 pacientes), religiosa (10 pacientes) e homeopatia (4 pacientes).

Médicos - Todos os 44 médicos disseram contar o diagnóstico ao paciente. A Tabela 2 mostra dados acerca do momento do diagnóstico na opinião dos médicos entrevistados. A maioria 35 (79,5\%) disse contar o diagnóstico quando este é confirmado. Vinte e quatro médicos $(54,6 \%)$ exigem a presença de um familiar no momento do diagnóstico. As res- 


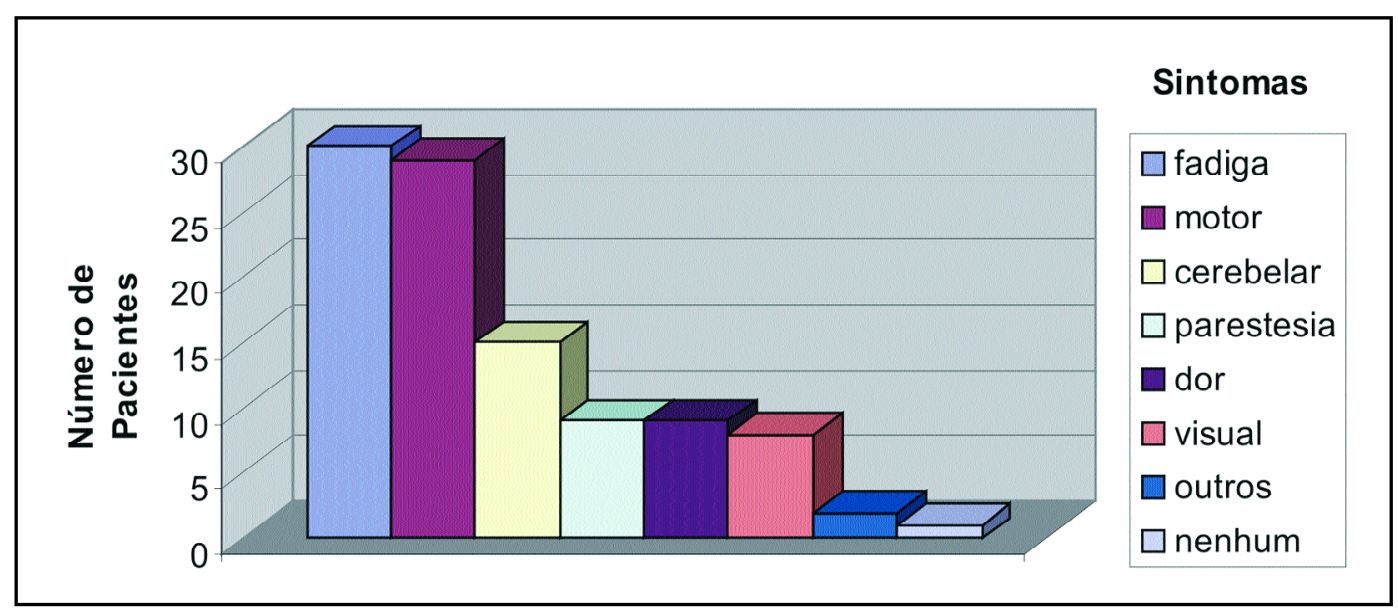

Gráfico 1. Sintomas relatados pacientes como sendo os que os mais the incomodam. Fonte: Centro de Atendimento e Tratamento de Esclerose Múltipla (CATEM) da Disciplina de Neurologia da Faculdade de Ciências Médicas da Santa Casa de São Paulo.

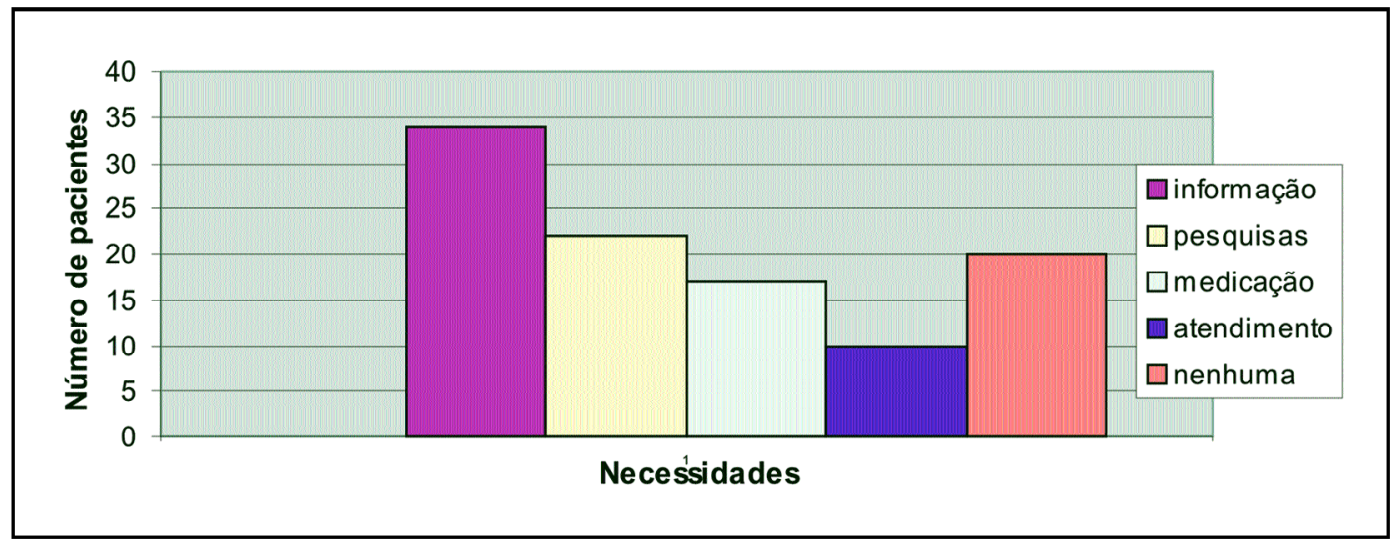

Gráfico 2. Necessidade dos pacientes para que a condução de seus casos fosse melhor.

postas quanto às orientações em uma possível gravidez foram variadas, sendo que 13 (29,6\%) médicos deram respostas genéricas e $9(20,4 \%)$ não responderam ou não souberam responder à questão (Ta bela 3). A maioria dos médicos afirmou que explica o tratamento realçando seus benefícios e efeitos colaterais $(59,1 \%)$. Outras respostas foram: de forma otimista (2,3\%); tirando as dúvidas dos pacientes $(25,0 \%)$; seguimento rigoroso $(4,5 \%)$; oferecendo a ele as opções, numa parceria (2,3\%); $6,8 \%$ não responderam.

Trinta e oito médicos $(86,4 \%)$ acreditam que a vida dos pacientes podia estar melhor. Este índice foi mais alto entre os assistentes $(94,4 \%)$ quando comparado aos dos residentes/estagiários (76,9\%). Trinta e cinco médicos $(79,5 \%)$ orientam sobre as limitações dos pacientes incentivando-os a levar uma vida o mais próximo do normal, conhecendo suas limitações e novamente, os assistentes tiveram um índice maior
( $88,9 \%$ vs. $69,2 \%)$. As opiniões a respeito das terapias complementares estão na Tabela 4.

Todos os médicos negaram terem tido pacientes que pediram para morrer.

\section{DISCUSSÃO}

O presente estudo apresenta diversas limitações. Primeiramente, houve muita relutância dos médicos em responder o questionário. Muitos recusaram a respondê-lo. Portanto, a amostra de médicos pode não ser representativa dos neurologistas. Segundo, o modo como as perguntas foram feitas, de forma aberta, sem opções pré-determinadas, embora permita saber melhor sobre a compreensão e os desejos dos pacientes e sobre a conduta dos médicos, dando um "diagnóstico" do problema, como é feito nas pesquisas qualitativas, dificulta a análise dos resultados, pois as respostas dos médicos e dos pacientes foram agrupadas segundo as impressões dos pes- 
quisadores. Terceiro, algumas perguntas feitas aos médicos, por apresentarem um conteúdo legal e moral, podem ter sido respondidas de forma "moralmente correta" e não segundo suas próprias opiniões. A mesma colocação, embora em menor grau, vale para a pergunta a respeito de terapias ditas alternativas feita aos pacientes.

Este trabalho mostrou que os pacientes querem saber seu diagnóstico, e a maioria acredita que o médico é quem lhe deve contá-lo. Um estudo anterior já confirmava este dado 9 . Conhecer seu diagnóstico é um direito do paciente, estabelecido pelo Código de Ética Médica. No nosso estudo, todos os médicos disseram contar o diagnóstico aos seus pacientes, baseados, muitos deles, na premissa anterior. Nelson ${ }^{8}$ e Sencer ${ }^{10}$ acreditam que não há nenhum benefício médico concreto em contar o diagnóstico em casos leves e iniciais. Alguns médicos ainda acreditam que a descoberta do diagnóstico poderia causar depressão e suicídio. Entretanto, esta suspeita não foi confirmada ${ }^{11}$. Outro ponto a ser considerado é a impotência que o médico sente quando se depara com uma condição ainda incurável $^{9}$. No entanto, o âmbito da Medicina são as necessidades humanas ${ }^{12}$ e para estas sempre há o que fazer.

A decisão de contar o diagnóstico deve levar em conta que o paciente necessita de um "nome" para sua doença, uma vez que ele pode ser rotulado de etilista ou doente mental devido aos sintomas que apresenta ${ }^{9}$. O médico, quando define um diagnóstico, deve pesar suas conseqüências, pois se trata de uma pessoa que está sofrendo ${ }^{13}$. O modo como o diagnóstico é contado é o mais importante ${ }^{14}$. Explicações e apoio contínuo são elementos essenciais nesta decisão ${ }^{15,16}$. Os pacientes que disseram ter sido errado o modo como o diagnóstico foi contado relataram que o mais incômodo foi o modo como o médico falou ("frio, nem se importando comigo", "como se não houvesse esperança" - nas palavras de duas pacientes) e, em seguida, a falta de explicações. A maioria dos médicos, ao ser indagada sobre as explicações dadas aos pacientes, disse explicar sobre a resposta do tratamento e suas complicações. Respostas como "tirando as dúvidas dos pacientes" ou "estabelecendo uma parceria" foram citadas por apenas $27,2 \%$ dos entrevistados.

Um familiar presente no momento de contar o diagnóstico tem importância especial para médicos e pacientes, devido ao maior entendimento que este pode ter da situação devido à situação de choque em que o paciente se encontra e ao apoio emocional que pode lhe dar ${ }^{8}$. No presente estudo, $47,7 \%$ dos médicos disseram exigir a presença de um familiar na consulta. No entanto, esta situação tem que ser autorizada pelo paciente, pois os segredo médico é essencial à profissão. A decisão de revelar o diagnóstico posteriormente só cabe ao paciente ${ }^{17}$. O médico deve, entretanto, orientar o paciente e afirmar que a confiança é a base das relações ${ }^{8}$. 0 momento de relatar o diagnóstico parece depender da relação que se estabeleceu entre médico e paciente. O médico deve, conhecendo seu paciente, decidir se o diagnóstico deve ser contado na suspeita clínica ou com a confirmação por exames complementares.

As perguntas em relação à gravidez e a hereditariedade da doença foram feitas no sentido de avaliar o conhecimento dos pacientes sobre a EM. Trinta e sete pacientes entrevistadas não desejavam ter filhos. Embora muitas relatassem uma escolha, citando principalmente o campo profissional, o número é ainda muito alto, mesmo quando se considera que a maior parte da amostra tem nível superior. Várias mulheres relataram que o fardo da doença pesou muito na decisão. Algumas opiniões foram: "O médico disse que na gravidez não há surtos, mas depois eles aparecem mais", "Parece que as medicações fazem mal à criança" e "Não sei como a doença vai progredir, não sei se poderia cuidar dos meus filhos". Atualmente, está bem documentado que a gravidez não interfere com a doença ${ }^{18}$, não devendo ser este um motivo para a desistência da maternidade. Logo, se este é um argumento citado na decisão, deve haver uma explicação errada por parte do médico. Quanto à progressão da doença, este é um motivo que deve ser levantado pelos possíveis pais, pois não há como prevê-la. O tratamento da EM envolve o uso de imunomoduladores e imunossupressores. Dentre os primeiros, o acetato de glatirâmer é seguro na gravidez. Os imunossupressores devem ser suspensos. As orientações dos médicos quanto à gravidez, segundo as respostas dadas, foram genéricas e muitas não estavam de acordo com o que é recomendado.

A maioria dos pacientes $(68,0 \%)$ negou que os filhos pudessem vir a ter a doença. Fatores genéticos claramente relacionam-se com a doença ${ }^{19,20}$, com risco em parentes de primeiro grau 20 a 40 vezes maior que na população em geral, o que significa, no entanto, um risco geral menor que $5 \%{ }^{19}$. Um risco pequeno, mas que deve ser do conhecimento do casal. Provavelmente, tal questão não foi adequadamente abordada nas consultas. 
Embora a incidência e a prevalência de doenças crônicas estejam aumentando, poucos estudos têm sido feitos para avaliar a qualidade de vida destes pacientes e de seus cuidadores ${ }^{21-24}$. Nosso estudo limitou-se a perguntar sobre o impacto da doença no paciente e o sintoma que mais Ihe incomodava, tendo uma abordagem apenas pontual do problema. Novos estudos são necessários para detalhar mais a qualidade de vida dos pacientes e pesquisar sobre os cuidadores e as pessoas que convivem com portadores de EM. Um estudo recente sobre o impacto das doenças crônicas no idoso diz que qualidade de vida nesta população é a "adaptação aos declínios da senescência, mantendo capacidade física, psíquica e de trabalho, e, quando necessário, também aprendendo a adaptar-se à doença"21. Esta definição cabe também aos pacientes com EM. As recomendações dos médicos da pesquisa seguiram esta definição. As orientações do médico são importantes neste sentido, mas uma abordagem multidisciplinar parece o meio mais efetivo de se alcançar este objetivo.

Informações sobre a doença são a maior necessidade dos pacientes. Como dito anteriormente, estas são importantes no momento em que o diagnóstico é declarado, mas também no estabelecimento da relação entre o paciente e o seu médico. Dentre as outras necessidades citadas, merecem destaque a medicação e o atendimento. A medicação para o tratamento da EM é fornecida pelo SUS e os pacientes que citaram este tópico relataram muita dificuldade em consegui-la. Quanto ao atendimento, os pacientes reclamaram da locomoção no ambulatório, citando a falta de cadeiras de rodas e os problemas com os elevadores. Um paciente levantou uma questão importante: "Acho que os pacientes mais graves deveriam ser atendidos em dias diferentes dos menos graves. Uma pessoa que acabou de receber o diagnóstico ao ver uma outra numa cadeira de rodas, sem falar direito, tremendo vai se assustar e pensar que também ficará assim, o que nem sempre é verdade".

A definição de terapias alternativas e complementares não é simples de ser feita. Aceita-se que elas sejam um conjunto de intervenções que não são ensinadas em escolas médicas ocidentais ou não estão geralmente disponíveis nos hospitais ${ }^{25}$. Recentemente, a acupuntura e a homeopatia foram reconhecidas como especialidades médicas, o que levou a mudança do termo para "terapia complementar". No nosso estudo, 27,2\% dos pacientes afirmaram fazer uso ou ter feito uso de alguma terapia com- plementar. Este número está próximo do britânico, e menor que o encontrado na França e na Alema$n h a^{26}$. Os estudos mostram que o uso de terapias alternativas é mais comum em doenças crônicas ${ }^{27}$. Ao contrário do que se pensa, não são situações de desespero que levam os pacientes a procurá-las, pois um número significante de pacientes já as procura para a manutenção da saúde ${ }^{25,28}$. O presente estudo não avaliou quando os pacientes procuraram a terapia complementar, nem seus motivos, mas todos referiram que o fizeram após a descoberta do diagnóstico. Antes dos médicos condenarem o uso de terapias complementares, como ocorreu no nosso estudo ( $50 \%$ dos médicos disseram, de alguma maneira, ser contrários ao uso destas terapias), devese entender porque os pacientes procuram-nas. Alguns dos motivos são a abordagem destas terapias e uma insatisfação com as atitudes dos médicos ${ }^{27} .0$ papel do médico quando o paciente opta por tal terapia é discutir com ele os motivos pelo qual ele fez esta escolha e mostrar as evidências ou falta delas para que o paciente possa fazer uma decisão baseada em informação. Para isto, o médico deve-se informar sobre elas. Discutir estes assuntos também enriquece a confiança na relação médico-paciente ${ }^{27}$.

Todos os médicos entrevistados negaram ter recebido pedidos para eutanásia ou suicídio assistido de pacientes com EM grave. Os médicos que ainda comentaram a questão relataram ser contrários devido à legislação brasileira proibir as duas práticas. Tal fato pode dever-se à cultura religiosa local que condena qualquer antecipação da morte. Não foi também encontrado nenhum artigo nacional que dê o posicionamento sobre estas questões. O mesmo não ocorre na literatura internacional ${ }^{29}$, em que são encontradas até diretrizes sobre o assunto ${ }^{30}$. No entanto, um estudo recente mostrou que os médicos, no caso neurologistas, não têm ainda conhecimento destas ${ }^{31}$, havendo uma grande diferença entre o que elas estabelecem e o que os médicos pensam. $O$ mesmo estudo mostra que se muitas das barreiras legais fossem removidas, muitos neurologistas participariam tanto da eutanásia quanto do suicídio assistido ${ }^{31}$. As taxas são realmente altas na Holanda, onde já existem leis que regulamentam tais procedimentos. Um estudo mostrou que $20 \%$ dos pacientes com esclerose lateral amiotrófica m orreram por suicídio assistido ou eutanásia ${ }^{32}$. No Brasil, a abreviação da vida do paciente por qualquer motivo não é permitida. A lei também não diferencia eutanásia "passiva" (por exemplo, a suspensão de antibióticos a um paciente terminal) da "ativa" (por exemplo, a administração de doses maiores de mor- 
fina, causando depressão respiratória) ${ }^{33}$. Assim, fazse necessária uma ampla discussão em nosso país sobre cuidados paliativos. No momento, a melhor solução talvez seja conversar previamente com o paciente e saber seus desejos, algo que só pode ser feito na parceria que representa a relação entre o paciente e seu médico.

Em conclusão, nosso estudo procurou dar um diagnóstico de como encontra-se a relação médicopaciente na EM. Assim, as principais conclusões que se fazem são: 1 . os pacientes querem saber seu diagnóstico e cabe ao médico informar-lhes; 2 . a forma de contar o diagnóstico é responsabilidade do médico e cabe a este, baseado no conhecimento que tem de seu paciente, escolher a forma e o momento de contá-lo, sempre oferecendo apoio ao doente; 3. os pacientes gostariam de mais informações sobre a sua doença; 4 . é necessário um debate sobre cuidados paliativos de pacientes com doenças crônicas, e; 5 . estudos posteriores são necessários para aprofundar algumas questões, como a qualidade de vida dos pacientes, seu grau de satisfação com o acompanhamento e seus desejos quanto aos cuidados paliativos.

\section{REFERÊNCIAS}

1. Blasco PG. O médico de família, hoje. São Paulo: SOBRAMFA, 1997.

2. Mc Cormick J. Death of personal doctor. Lancet 1996;348:667-668.

3. Morgenstern L. Will the real doctor please stand up? JAMA 2003;289:298

4. Sweeney KG, MacAuley D, Gary DP. Personal significance: the third dimension. Lancet 1998;351:134-136.

5. Cohen C, Marcolino JAM. Relação médico-paciente: autonomia \& paternalismo In Cohen C, Segre M. (EDS) Bioética. 2 Ed. São Paulo: Edusp, 1999:51-62.

6. Moreira MA, Felipe E, Mendes MF, Tilbery CP. Esclerose múltipla: estudo descritivo de suas formas clínicas em 302 casos. Arq Neuropsiquiatr 2000;58:460-466.

7. NoseworthyJH, Lucchinetti C, Rodriguez M, Weinshenker BG. Multiple sclerosis. N Engl J Med 2000;343:938-952.

8. Nelson RF. Ethical issues in multiplesclerosis. Semin Neurol 1997;17:227234.

9. Elian M, Dean G. To tell or not to tell the diagnosis of multiple sclero sis. Lancet 1985;2:27-28.

10. Sencer W. Suspicion of multiplesclerosis: to tell or not to tell? Arch Neurol 1988;45:441-442.

11. Bovermann M. Truth telling in medicine. JAMA 1982; 248:248.
12. Phillips WR, Haynes DG. The domain of family practice: scope, role, and function. Fam Med 2001;33:273-277.

13. Kleinman A, Eisenberg L, Good B. Culture, illness, and care: clinical lessons from anthropologic and cross-cultural research Ann Intern Med 1978;88:251-258.

14. Hachinski V. Suspected multiple sclerosis: how much to tell the patient? Arch Neurol 1988;45:442.

15. Calman K. The profession of medicine. BMJ 1994;309:1140-1143.

16. Price J, Leaver L. ABC of psychological medicine: beginning treatment. BMJ 2002;325:33-35

17. Cohen C. Confidencialidade: questões éticas relativas ao segredo profi issional. In Cohen C, Segre M - (EDS) Bioética. 2.Ed. São Paulo: Edusp, 1999.

18. Sadovnick AD, Eisen K, Hashimoto SA, et al. Pregnancy and multiple sclerosis: a prospective study. Arch Neurol 1994;51:1120-1124.

19. Sadovnick AD, Baird PA, Ward RH. Multiple sclerosis: updated risks for relatives. Am J Med Genet 1988;29:533-541.

20. Chataway J, Feakes R, Coraddu F, et al. The genetics of multiple sclerosis: principles, background and updated results of the United Kingdom systematic genome screen. Brain 1998; 121:1869-1887.

21. Cação JC, Souza AC, Aragaki GN, Takahashi ME, Souza PT, Guimarães RGR. Repercussões da doença crônica na qualidade de vida de pacientes idosos ambulatoriais. Rev Bras Clin Terap 2002;28:181-184.

22. Chagas Medeiros MM, Ferraz MB, Quaresma MR. The effect of rheumatoid arthritis on the quality of life of primary caregivers. J Rheumatol 2000;27:76-83.

23. Calman KC. Quality of life in cancer patients: an hypothesis. J Med Ethics 1984;10:124-127.

24. Pereira EDB. Qualidade de vida dos pacientes com doença pulmonar obstrutiva crônica e de seus cuidadores primários. Site:www.pneumoatu al.com.br (Acessado em 30/11/2002)

25. Eisenberg DM, Kessler RC, Foster C, NorlockFE, Calkins DR, Delbanco TL. Unconventional medicine in the United States. Prevalence, costs, and patterns of use. N Engl J Med 1993;328:246-252.

26. Fisher P, Wa rdA. Complementary medicine in Europe. BMJ 1994;309:107111.

27. Pappas S, Perlman A. Complementary and alternative medicine: the importance of doctor-patient communication. Med Clin NAm 2002;86:1-10.

28. Astin JA. Why patients use alternative medicine: results of a national study. JAMA 1998;279:1548-1553.

29. Meier DE, Emmons CA, Wallenstein S, Quill T, Morrison RS, CasselCK. A national survey of physician-assisted suicide and euthanasia in the United States. N Engl J Med 1998;338:1193-1201.

30. Report of the Ethics and Humanities Subcommittee of the American Academy of Neurology, Position statement: assisted suicide, euthanasia, and the neurologist. Neurology 1998 50:596-598.

31. Carver AC, Vickrey BG, Bernat JL, Keran C, Ringel SP, Foley KM. Endof-life care: a survey of US neurologists' attitudes, behavior, and knowledge. Neurology 1999;53:284-293.

32. Veldink JH, Wokke JH, van der Wal G, Vianney de Jong JM, van den Berg LH. Euthanasia and physician-assisted suicide among patients with amyotrophic lateral sclerosis in the Netherlands. N Engl J Med 2002; 346:1638-1644.

33. Segre M. Limites éticos da intervenção sobre o Ser humano In Cohen C, Segre M (EDS). Bioética. 2.Ed. São Paulo: Edusp, 1999:101-115. 